\title{
Surface Exciton-Plasmon Polariton Enhanced Light Emission via Integration of Single Semiconductor Nanowires with Metal Nanostructures
}

\author{
Xuejin Zhang ${ }^{1}$, Pengwei Wang ${ }^{1}$, Xinzheng Zhang ${ }^{1}$, Jun $\mathrm{Xu}^{1}$, Yongyuan Zhu ${ }^{2}(\varangle)$, and Dapeng Yu ${ }^{1}(\varangle)$ \\ ${ }^{1}$ State Key Laboratory for Mesoscopic Physics, and Electron Microscopy Laboratory, Department of Physics, Peking University, Beijing \\ 100871, China \\ ${ }^{2}$ National Laboratory of Solid State Microstructures, Nanjing University, Nanjing 210093, China \\ Received: 12 October 2008 / Accepted: 9 November 2008 \\ CTsinghua University Press and Springer-Verlag 2009. This article is published with open access at Springerlink. com
}

\begin{abstract}
The light emission enhancement behavior from single $\mathrm{ZnO}$ nanowires integrated with metallic contacts is investigated by micro-photoluminescence measurements. Apart from surface plasmon polaritons at the air/ metal interface, the emission of a single $\mathrm{ZnO}$ nanowire can be coupled into guided modes of surface excitonplasmon polaritons (SEPPs). The out-coupling avenues of SEPP guided modes are modeled in the presence of nanostructures, such as corrugation and gratings, on the metal surface. The guided modes of SEPPs in metalcontacted $\mathrm{ZnO}$ nanowires are calculated using the effective index method. The enhanced light emission from single semiconductor nanowires shows promise for use in highly efficient nano-emitters and nano-lasers, as well as macroscopic solid state light sources with very high efficiency.
\end{abstract}

\section{KEYWORDS}

Semiconductor nanowires, surface plasmon polaritons, nanowire waveguides, emission enhancement, gratings

Electromagnetic (EM) energy can be confined and guided at interfaces by virtue of surface polaritons, where light-matter interactions take place. Such bounded waves decay away from the interface into both media and also propagate along the interface with very large wave vectors compared with those in free space. The resonant interactions between the surface charge oscillations and the EM field of the electromagnetic waves constitute the surface plasmon polaritons (SPPs), which underlie a variety of fascinating phenomena, such as surface enhanced Raman scattering [1], extraordinary transmission of light through subwavelength holes in metal films [2], and superlens effects with sub-diffractionlimited resolution [3]. The properties of SPPs can be tailored by introducing periodic nanostructures at the interface, which provide possibilities for new types of photonic devices. As a new branch of photonics, plasmonics based on SPPs is being explored for its potential applications in diverse fields, such as subwavelength optics, data storage, light generation, solar cells, microscopy, and bio-photonics [4].

New generation solid state light emitters such as light-emitting diodes (LEDs) are expected to

Address correspondence to Dapeng Yu, yudp@pku. edu. cn; Yongyuan Zhu, yyzhu@nju. edu. cn 
replace traditional light sources, which suffer from their limited light emission efficiency. For example, only about $2 \%$ of the light in a conventional LED can be extracted from a narrow cone $\sim 18^{\circ}$ normal to the surface. Several techniques, including resonant cavities [5], photonic crystal structures [6], and SPPs [7-10], have been developed to enhance both the internal quantum efficiency and the light extraction efficiency.

On the other hand, as nano-emitters, nanowires tend to generate polarized light, which may have a wider range of applications. For example, the efficiency of a nanowire-based laser can be increased and the threshold current intensity can be decreased due to polarization. If the nanowires are aligned parallel on a chip, polarized LEDs and planar displayers can be developed. Photodetection based on single InP nanowires with highly polarized photoluminescence (PL) [11] and electrically driven LEDs/laser diodes based on single CdS nanowires [12] have been demonstrated. If highly efficient nanowire light-emitters can be realized, devices based on nanowires would be more promising. Effective emission enhancement for a single semiconductor nanowire is a prerequisite for future light-emitting devices. In this paper, we address this issue by means of integrating single $\mathrm{ZnO}$ nanowires with metallic contacts. In this case, the exciton emission in $\mathrm{ZnO}$ nanowires in the ultraviolet region will couple strongly with the surface charge oscillation in the metal, resulting in guided modes of a novel surface polariton. Two kinds of nanostructures, roughness and gratings on a metal surface, are demonstrated to effectively out-couple the resultant surface polaritons.

The monocrystalline $\mathrm{ZnO}$ nanowires were synthesized by the vapor-liquid-solid
(VLS) method [13] and then dispersed on a quartz substrate. Single $\mathrm{ZnO}$ nanowires were partially coated by $40 \mathrm{~nm}$ thick Ag film patches using electron beam lithography (EBL) and magnetron sputtering. The light emission from a $\mathrm{ZnO}$ nanowire was investigated using micro-photoluminescence ( $\mu$-PL) spectroscopy at room temperature. A He-Cd laser at $325 \mathrm{~nm}$ with a spot diameter $\sim 2 \mu \mathrm{m}$ was focused onto the single $\mathrm{ZnO}$ nanowire from the other side of the substrate during $\mu$-PL measurements, as shown in Fig. 1(a). For comparison, the experiment was repeated for the same single $\mathrm{ZnO}$ nanowire with, and without, Ag contact. Scanning electron microscopy (SEM) shows that the single $\mathrm{ZnO}$ nanowire is uniform along its axis with a rectangular cross section (shown in Fig. 1(b)). Atomic force microscopy (AFM) confirms a square cross section, whose length of side is about $106 \mathrm{~nm}$. Transmission electron microscopy (TEM) analysis shows that the $\mathrm{ZnO}$ nanowire did not grow exactly along the $c$-axis, but at a certain angle, which is in accordance with the non-hexangular cross section of the single $\mathrm{ZnO}$ nanowire.

Figures $1(\mathrm{c})$ and $1(\mathrm{~d})$ show the $\mu$-PL spectra of the single $\mathrm{ZnO}$ nanowires which have two characteristic

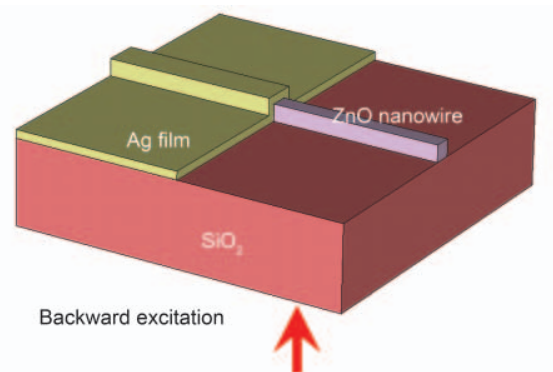

(a)

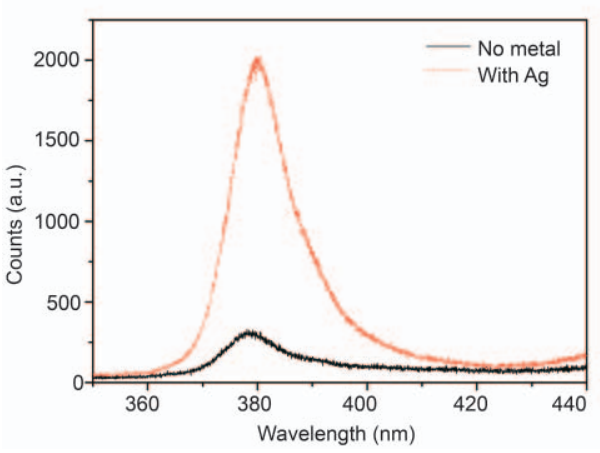

(c)

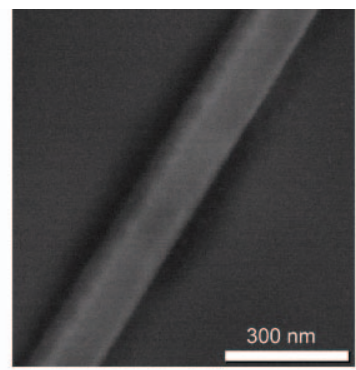

(b)

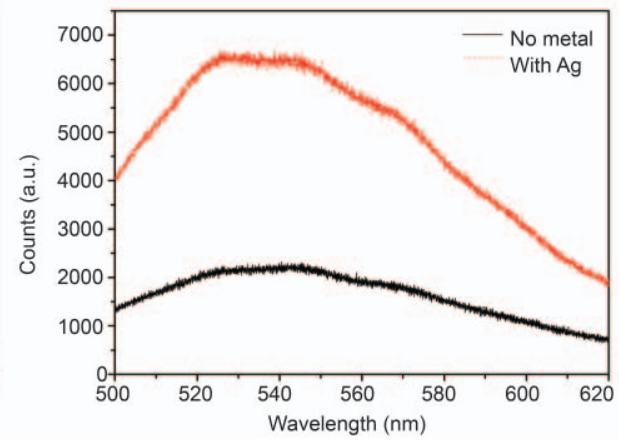

(d)

Figure 1 (a) Schematic configuration for $\mu$-PL measurements; (b) SEM image of a single ZnO nanowire. Room temperature $\mu$-PL spectra in (c) ultraviolet and (d) green regions taken from a single ZnO nanowire without (black solid line) and with (red dotted line) Ag contact 
emission peaks in the ultraviolet $(\sim 378 \mathrm{~nm})$ and green $(\sim 530 \mathrm{~nm})$ regions. The green emission is usually attributed to impurities in $\mathrm{ZnO}$ nanowires, which is more intense than that of the exciton emission for our sample. The emission intensities with Ag contacts are higher than those without Ag contacts under the same excitation conditions. The enhancement ratio of peak intensities with/without $\mathrm{Ag}$ contacts is $\sim 6$ for the ultraviolet (Fig. 1(c)) and $\sim 3$ for the green (Fig. 1(d)) emission bands.

The enhancement can be improved by optimization of the structure based on the nanowires. In the case of SPP-mediated emission, a photon emitted from the active source can transfer into an SPP mode instead of into free space. If band gap structures of SPPs are introduced via photonic crystal structures, the density of states (DOS) of SPPs can be increased considerably at the band edges [14]. In addition, when periodic nanostructures are introduced, the out-coupling of SPPs is expected to be more effective than the case of introducing metal surface roughness. Here, instead of $\mathrm{Ag}$ (if $\mathrm{Ag}$ is used, the period will be too small), one-dimensional (1-D) Al gratings were integrated with a single $\mathrm{ZnO}$ nanowire (shown in Fig. 2(a)), where $25 \mathrm{~nm}$ thick 1-D Al gratings with a periodicity $\sim 500 \mathrm{~nm}$ were coated on a silicon substrate fabricated using EBL and magnetron sputtering. A single $\mathrm{ZnO}$ nanowire with length $\sim 17 \mu \mathrm{m}$ and transverse dimension twice that of the previous one was dispersed onto the 1-D Al gratings. Figure 2(b) shows the $\mu$-PL spectra in the ultraviolet region collected from the $\mathrm{ZnO}$ nanowire with and without the 1-D Al gratings. The enhancement ratio of the $\mu$-PL peak heights with/without the metal is $~ 17$ while that of the integrated peak intensity reaches 21 , with a red-shift of $\sim 7 \mathrm{~nm}$.

A possible mechanism for the out-coupling process of the emission is shown in Fig. 3. Apart from directly radiating photons into free space from the nanowire, as for the case of a single $\mathrm{ZnO}$ nanowire without metallic contact, there are several other radiation pathways for a $\mathrm{ZnO}$ nanowire with metallic contact. Arrows 1 and 2 denote the processes in which the emission is first coupled into SPPs at the air/metal interface and the resulting SPPs are out-coupled by the

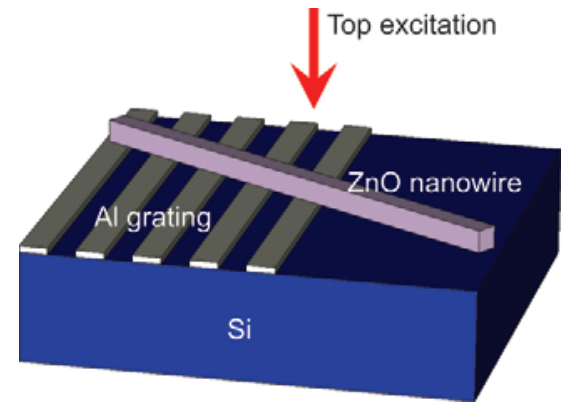

(a)

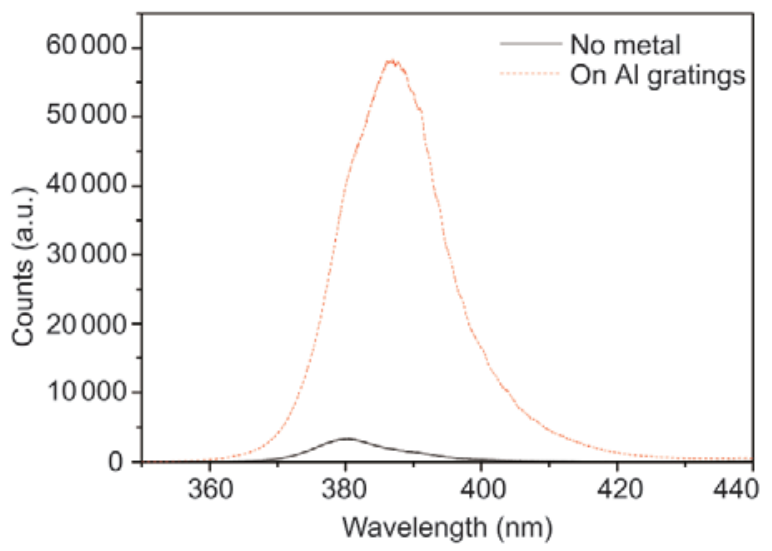

(b)

Figure 2 (a) Schematic configuration for $\mu$-PL measurements; (b) room temperature $\mu$-PL ultraviolet spectra of a single $\mathrm{ZnO}$ nanowire without (black solid line) and with (red dotted line) Al gratings

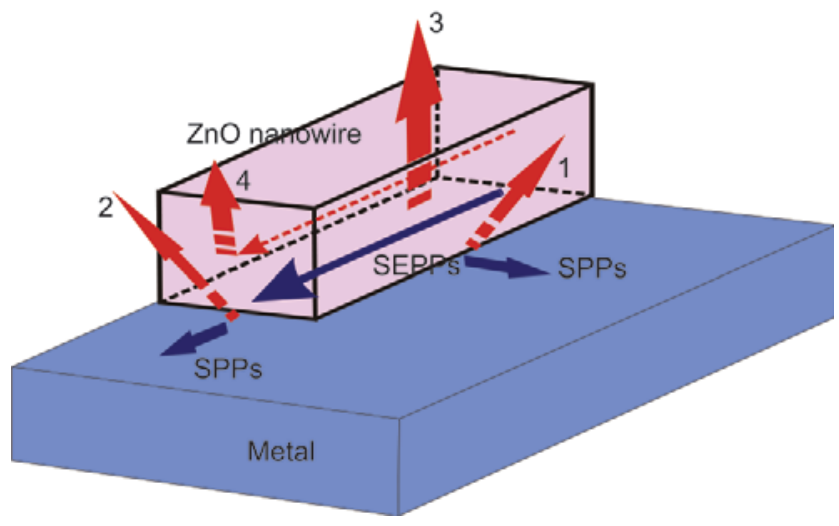

Figure 3 Schematic of the out-coupling mechanism of SEPPS at the metal/ZnO nanowire interface and SPPs at the surrounding metal/ dielectric interface. The blue arrows represent the surface bound nonradiative modes while the red striped arrows the radiative modes out-coupled by the nanostructures on the metal surface. The dashed arrow along the axis of the $\mathrm{ZnO}$ nanowire denotes the non-SEPP modes in the $\mathrm{ZnO}$ nanowire waveguide 
nanostructures, such as roughness or gratings on the metal surface. On the other hand, the resonant interaction between the surface charge oscillations in the metal and the EM field of the excitonpolaritons in the $\mathrm{ZnO}$ nanowire plays an important role in the luminescence in the ultraviolet region. We denote the quantum of the coupled wave field as a surface exciton-plasmon polariton (SEPP). The emission can also be converted into guided modes of SEPPs in a $\mathrm{ZnO}$ nanowire, which can be converted to leaky modes and other non-SEPP modes of a waveguide, out-coupled as shown in processes 3 and 4 . More details will be explained below.

By virtue of the small size, a large portion of the evanescent field of the emitted light is outside a single $\mathrm{ZnO}$ nanowire. Thus, the emission from the nanowire will be coupled laterally into the SPPs of surrounding metal/dielectric interfaces, and can be reemitted via the out-coupling of the SPPs owing to the roughness or gratings on the metal surfaces. The inset of Fig. 4(a) shows the roughness of Ag surface as observed by SEM (grain size $\sim 40 \mathrm{~nm}$ ). Figure 4(a) shows the dispersion relation of SPPs at the air/Ag/ $\mathrm{SiO}_{2}$ interfaces with $40 \mathrm{~nm}$ thick $\mathrm{Ag}$, which is mapped by transmissivity according to Fresnel's equations [15]. In our calculations, the dielectric functions of $\mathrm{Ag}, \mathrm{Al}, \mathrm{ZnO}, \mathrm{SiO}_{2}$, and $\mathrm{Si}$ are taken from experimental data $[16,17]$, and the material loss is neglected. There exist two branches of the SPPs. The upper branch is mainly for the air/Ag interface, while the lower one is mainly for the $\mathrm{Ag} / \mathrm{SiO}_{2}$ interface. The two branches of SPPs are coupled as a result of the finite thickness of Ag. The SPP resonant wavelength (corresponding
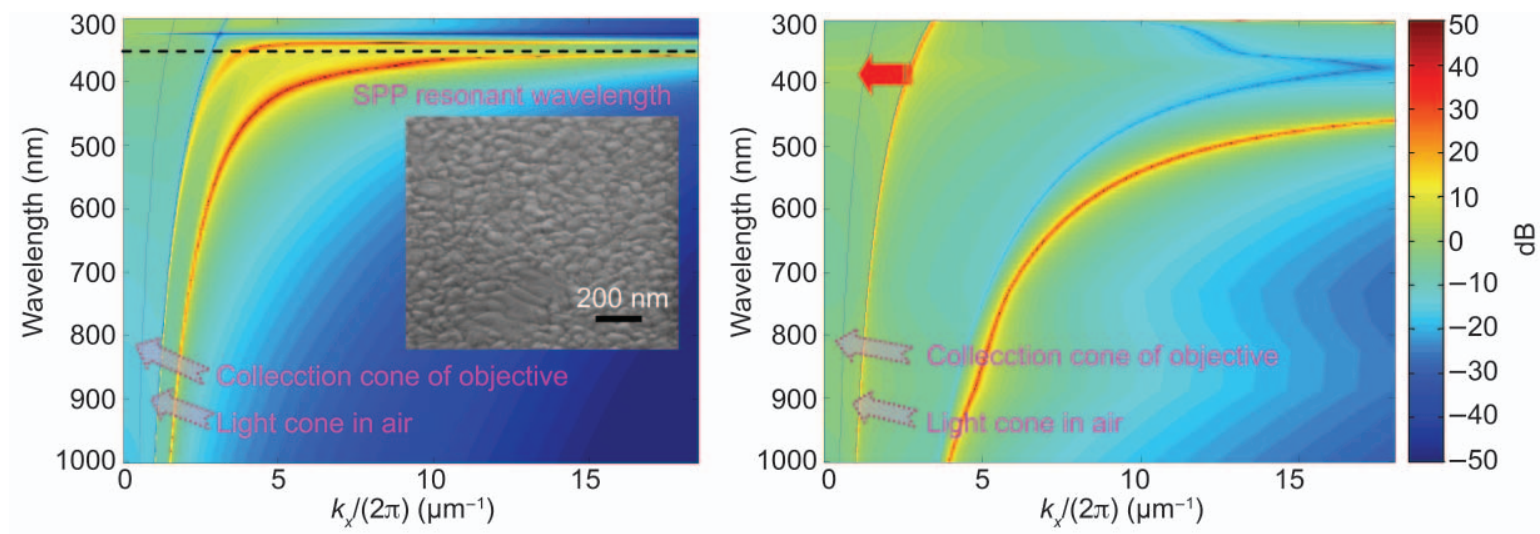

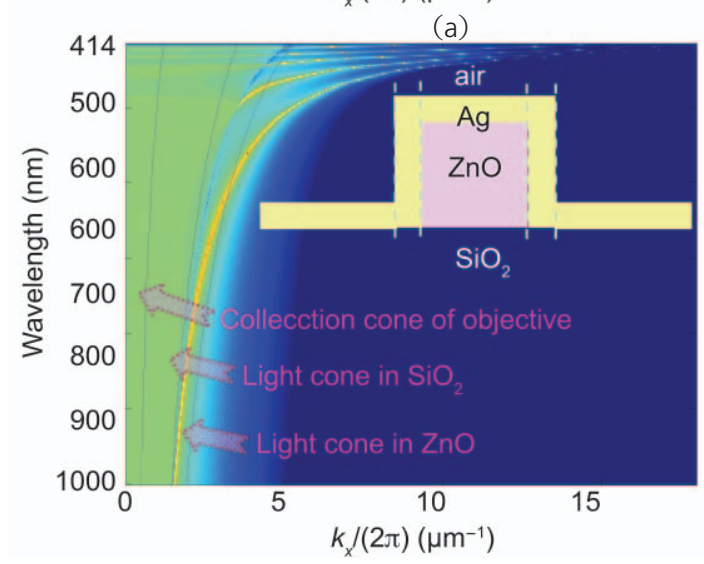

(c)

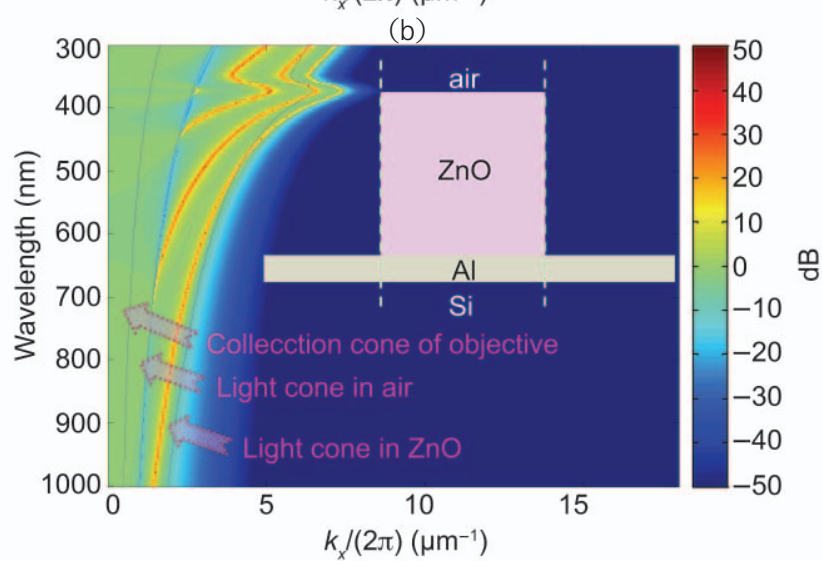

(d)

Figure 4 The dispersion relation of the SPPs at the (a) air/Ag/ $\mathrm{SiO}_{2}$ and (b) air/Al/Si interfaces with $40 \mathrm{~nm}$ thick $\mathrm{Ag}$ and $25 \mathrm{~nm}$ thick Al, respectively. The dashed horizontal line in (a) represents the position of the SPP resonant wavelength for the $\mathrm{Ag} / \mathrm{SiO} \mathrm{S}_{2}$ interface. An SEM image of the surface morphology of the Ag film is shown as an inset in (a). The top arrow in (b) denotes an out-coupling process of SPPs from the air/Al interface to the collection cone of the objective. The SEPP-related fundamental guided modes of (c) a Ag-contacted single $\mathrm{ZnO}$ nanowire (inset) with side length of $106 \mathrm{~nm}$ and (d) an Al-contacted single $\mathrm{ZnO}$ nanowire (inset) with side length of $216 \mathrm{~nm}$ as calculated by the effective index method 
to the largest SPP-DOS) of the lower branch is about $360 \mathrm{~nm}$ and, therefore, the SPP coupling will be more effective at wavelengths closer to $360 \mathrm{~nm}$. The larger SPP-DOS near the resonant wavelength and the very large wave vectors afforded by roughness sustain the larger enhancement ratio for the ultraviolet band than that for the green band, as shown in Figs. 1(c) and $1(\mathrm{~d})$, even though the green emission is more intense than the ultraviolet emission. Figure 4(b) shows the SPP dispersion relationship of the air/Al/Si interfaces with $25 \mathrm{~nm}$ thick Al. The left branch corresponds mainly to the air/Al interface and the right branch to the $\mathrm{Al} / \mathrm{Si}$ interface, and they are coupled with each other. It can be seen that the left branch is very close to the light path in air, so that the SPPs are prone to radiate into the collection objective in response to the momentum conservation: $(2 \pi / \lambda) \sin \left(\theta_{m}\right)=\operatorname{Re}\left\{k_{\mathrm{SPP}}(\lambda)\right\}+$ $m G_{0}$. Here $\theta_{m}$ is the angle of emission, $m$ is an integer and $G_{0}=2 \pi / \Lambda$, where $\Lambda$ is the grating period. Due to the momentum conservation, the above-observed enhancement in PL emission results mainly from the 1-D Al nanogratings.

Taking into account the three-dimensional nature of the system, we calculate the dispersion relation using the effective index method [18], which has an accuracy within $1 \%$ when dealing with fundamental modes [19]. The guided modes related to SEPPs are transverse magnetic (TM)-like modes. The fundamental TM-like modes of the single $\mathrm{ZnO}$ nanowire with the configuration of Fig. 1(a) are mapped in Fig. 4(c). The TM modes in the multilayer structures air $/ \mathrm{Ag} / \mathrm{ZnO} / \mathrm{SiO}_{2}$ and $\operatorname{air} / \mathrm{Ag} / \mathrm{SiO}_{2}$ were first calculated. To be consistent with our experimental configuration, the effective indices of SEPP modes guided along the $\mathrm{Ag} / \mathrm{ZnO} / \mathrm{SiO}_{2}$ interface and SPP modes at the $\mathrm{Ag} / \mathrm{SiO}_{2}$ interface are used for the inner and outer layers of the waveguide structure, shown as an inset in Fig. 4(c). From Fig. 4(c), the peak position in the ultraviolet is beyond the SEPP resonant wavelength, and only the lowest order SEPP mode acts on the emission enhancement at $530 \mathrm{~nm}$. It should be noted that material loss can be involved in the emission enhancement of $\mathrm{ZnO}$ nanowires by means of quasi-bound modes and radiative modes (group velocity $v_{\mathrm{g}}<0$ ) [20] with large DOS above the SEPP resonant wavelength.
The effective index method is also employed to calculate the guided modes of SEPPs along the axis of a $\mathrm{ZnO}$ nanowire with a similar structure to that shown in Fig. 2(a), where the $25 \mathrm{~nm}$ thick 1-D $\mathrm{Al}$ grating is replaced by a $25 \mathrm{~nm}$ thick $\mathrm{Al}$ film. The main TM-like modes of the single $\mathrm{ZnO}$ nanowire are mapped in Fig. 4(d). The TM modes for the multilayer structures air/ZnO/Al/Si and air/Al/Si were first calculated. The effective indices of the SEPP modes guided along $\mathrm{ZnO} / \mathrm{Al}$ and SPP modes at air/Al interfaces are used for the inner and outer layers of the waveguide structure, respectively, as shown in the inset in Fig. 4(d), which is in accordance with our experimental configuration. The drastic change in dispersion of SEPP modes reflects the strong coupling between the photon and exciton in the $\mathrm{ZnO}$ nanowire. Compared to that of $\mathrm{ZnO}$ and air, the dispersion curves of the guided modes of SEPPs possess larger DOS, especially in the ultraviolet spectral region, leading to a high emission rate into the SEPP modes, determined by Fermi's golden rule. The waveguide modes can propagate several times along the axis of the $\mathrm{ZnO}$ nanowire. The larger the excitation density, the longer the waveguide modes propagate, and thus the more out-coupling the processes shown in Fig. 3. The red-shift effect in Fig. 2(b) can be understood when considering the propagation of guided modes and the reabsorption/reemission process of exciton emission. In addition, the FranzKeldysh effect [21] will also contribute to the red -shift in the manner of the strong electric field of SEPPs on a $\mathrm{ZnO} /$ metal surface. The $\mathrm{ZnO}$ nanowire is expected to be pumped and generate gain, even before the threshold for lasing, with increasing excitation density. Hence, the waveguide effect in $\mathrm{ZnO}$ nanowires is substantial for highly efficient light-emitting devices.

The above SEPP/SPP-mediated emission scheme can be extended to other semiconductor nanowires. The emission enhancement originates from the competitive spontaneous emission conversion between the SEPP/SPP modes and the radiative/nonradiative photon modes. A large SEPP/SPP-DOS, arising from the well-designed dispersion relation of SEPPs/SPPs, will result in a high spontaneous

\section{量 Springer}


emission rate into the SEPP/SPP modes, which can be effectively extracted via micro/nanostructures. The SEPP/SPP-enhanced light emission becomes facile in electroluminescence devices, because the metal contact can bear SEPPs/SPPs, as well as electrical currents. The SEPPs also harbor great promise for appreciable lower lasing thresholds of a single nanowire. Our results shed light on the fabrication of highly efficient nano-emitters and nano-lasers, pivotal components in photonic circuits at nanoscale dimensions.

\section{Methods}

The $\mathrm{ZnO}$ nanowires were synthesized by the traditional VLS method [13]. A mixture of $\mathrm{ZnO}$ powder and graphite with a molar ratio 1:1 was placed in a ceramic boat, which lay in the center of a horizontal tube furnace. The whole system was heated to $1050{ }^{\circ} \mathrm{C}$ in $35 \mathrm{~min}$ under a constant flow of 200 standard cubic centimeters per minute $(\mathrm{sccm}) \mathrm{Ar}$ and then kept at $1050{ }^{\circ} \mathrm{C}$ for 30 min under a constant flow of a mixture of $200 \mathrm{sccm}$ Ar and 2. $6 \mathrm{sccm} \mathrm{O}_{2}$ under atmospheric pressure. The furnace was cooled to room temperature naturally under Ar flow. The $\mathrm{ZnO}$ nanowires were collected on a $2 \mathrm{~nm}$ thick Aucoated silicon (111) substrate, $2 \mathrm{~cm}$ downstream of the gas flow to the source. The crystal structure of the $\mathrm{ZnO}$ nanowires was determined by high-resolution TEM.

All metallic structures, including markers, patches, and gratings, were fabricated by EBL. Polymethylmethacrylate (PMMA) was diluted in anisole (4 $\mathrm{wt} \%$ ) and used as a positive resist for EBL. The $\mathrm{ZnO}$ nanowires were suspended in ethanol and well spread on a fused quartz substrate with Ag markers, by which individual nanowires were located. The single $\mathrm{ZnO}$ nanowires were partially coated by $40 \mathrm{~nm}$ thick Ag film patches by EBL, development of the resist, magnetron sputtering, and lift-off procedures. $25 \mathrm{~nm}$ thick Al gratings with the periodicity of $500 \mathrm{~nm}$ were fabricated by EBL on the silicon substrate before $\mathrm{ZnO}$ nanowires were dispersed onto it. The size and morphology of the $\mathrm{ZnO}$ nanowires and metallic structures were investigated by SEM and AFM.
$\mu$-PL measurements were performed by using a Renishaw inVia Raman microscope. The excitation wavelength was $325 \mathrm{~nm}$ with a He-Cd laser, which was focused onto the single $\mathrm{ZnO}$ nanowire with a spot diameter $\sim 2 \mu \mathrm{m}$, using an optical microscope objective (40×magnification, NA 0.5). During $\mu$-PL measurements, the excitation densities were $\sim 150$ $\mathrm{kW} / \mathrm{cm}^{2}$ for Ag-contacted samples and $\sim 15 \mathrm{~kW} / \mathrm{cm}^{2}$ for Al-contacted samples.

\section{Acknowledgements}

This work was financially supported by the National Natural Science Foundation of China (NSFC, Nos. 90606023, 10574003, 10523001), a joint project with Research Grants Council of Hong Kong (NSFC/ RGC 20731160012), and national key projects (2002CB613505, 2007CB936202, MOST). D. P. Yu is supported by the Cheung Kong Scholar Program, Ministry of Education, P. R. China.

\section{References}

[1] García-Vidal, F. J.; Pendry, J. B. Collective theory for surface enhanced Raman scattering. Phys. Rev. Lett. 1996, 77, 1163-1166.

[2] Ebbesen, T. W.; Lezec, L. J.; Chaemi, H. F.; Thio, T.; Wolff, P. A. Extraordinary optical transmission through subwavelength hole arrays. Nature 1998, 391, 667-669.

[3] Fang, N.; Lee, H.; Sun, C.; Zhang, X. Sub-diffractionlimited optical imaging with a silver superlens. Science 2005, 308, 534-537.

[4] Barnes, W. L.; Dereux, A.; Ebbesen, T. W. Surface plasmon subwavelength optics. Nature 2003, 424, 824-830.

[5] Purcell, E. M. Spontaneous emission probabilities at radio frequencies. Phys. Rev. 1946, 69, 681.

[6] Diana, F. S.; David, A.; Meinel, I.; Sharma, R.; Weisbuch, C.; Nakamura, S.; Petroff, P. M. Photonic crystal-assisted light extraction from a colloidal quantum dot / GaN hybrid structure. Nano Lett. 2006, 6, 1116-1120.

[7] Okamoto, K.; Niki, I.; Shvartser, A.; Narukawa, Y.; Mukai, T.; Scherer, A. Surface-plasmon-enhanced light emitters based on InGaN quantum wells. Nat. Mater. 2004, 3, 601-605.

[8] Neogi, A.; Lee, C. -W.; Everitt, H. O.; Kuroda, T.; Tackeuchi, A.; Yablonovitch, E. Enhancement of 
spontaneous recombination rate in a quantum well by resonant surface plasmon coupling. Phys. Rev. B 2002, 66, 153305.

[9] Gontijo, I.; Boroditsky, M.; Yablonovitch, E.; Keller, S.; Mishra, U. K.; DenBaars, S. P. Coupling of InGaN quantum-well photoluminescence to silver surface plasmons. Phys. Rev. B 1999, 60, 11564-11567.

[10] Okamoto, K.; Niki, I.; Scherer, A.; Narukawa, Y.; Mukai, T.; Kawakami, Y. Surface plasmon enhanced spontaneous emission rate of InGaN/GaN quantum wells probed by time-resolved photoluminescence spectroscopy. Appl. Phys. Lett. 2005, 87, 071102.

[11] Wang, J.; Gudiksen, M. S.; Duan, X.; Cui, Y.; Lieber, C. M. Highly polarized photoluminescence and photodetection from single indium phosphide nanowires. Science 2004, 293, 1455-1457.

[12] Duan, X.; Huang, Y.; Agarwal, R.; Lieber, C. M. Singlenanowire electrically driven lasers. Nature 2003, 421, 241-245.

[13] Wagner, R. S.; Ellis, W. C. Vapor-liquid-solid mechanism of single crystal growth. Appl. Phys. Lett. 1964, 4, 8990.

[14] Zhang, J.; Ye, Y. -H.; Wang, X.; Rochon, P.; Xiao, M. Coupling between semiconductor quantum dots and two-dimensional surface plasmons. Phys. Rev. B 2005, 72, 201306.

[15] Zhang, X. J.; Wu, D. M.; Sun, C.; Zhang, X. Artificial phonon-plasmon polariton at the interface of piezoelectric metamaterials and semiconductors. Phys. Rev. B 2007, 76, 085318.

[16] Yoshikawa, H.; Adachi, S. Optical constants of ZnO. Jpn. J. Appl. Phys. 1997, 36, 6237-6243.

[17] Palik, E. D. Handbook of Optical Constants of Solids; Academic Press: Orlando, 1985.

[18] Marcuse, D. Theory of Dielectric Optical Waveguides; Academic Press: San Diego, 1991.

[19] Karalis, A.; Lidorikis, E.; Ibanescu, M.; Joannopoulos, J. D.; Soljačić, M. Surface-plasmon-assisted guiding of broadband slow and subwavelength light in air. Phys. Rev. Lett. 2005, 95, 063901.

[20] Dionne, J. A.; Sweatlock, L. A.; Atwater, H. A.; Polman, A. Planar metal plasmon waveguides: Frequency-dependent dispersion, propagation, localization, and loss beyond the free electron model. Phys. Rev. B 2005, 72, 075405.

[21] Cavallini, A.; Polenta, L.; Rossi, M.; Stoica, T.; Calarco, R.; Meijers, R. J.; Richter, T.; Lüth, H. Franz-Keldysh effect in GaN nanowires. Nano Lett. 2007, 7, 2166-2170. 